hep-th/0407043

SCIPP-2004/11

SU-ITP-04-28

\title{
Low Energy Supersymmetry From the Landscape
}

\author{
Michael Dine and Elie Gorbatov \\ Santa Cruz Institute for Particle Physics, Santa Cruz CA 95064 \\ Scott Thomas \\ Physics Department, Stanford University, Stanford, CA 94305
}

\begin{abstract}
There has been some debate as to whether the landscape does or does not predict low energy supersymmetry. We argue that under rather mild assumptions, the landscape seems to favor such breaking, quite possibly at a very low scale. Some of the issues which must be addressed in order to settle these questions are the relative frequency with which tree level and non-perturbative effects generate expectation values for auxiliary fields and the superpotential, as well as the likelihood of both $R$ - and non- $R$ discrete or accidental symmetries. Alternative scenarios with warped compactifications or large extra dimensions are also discussed.
\end{abstract}




\section{Introduction: Supersymmetry and the Landscape}

Supersymmetry and/or technicolor have long been advocated as possible solutions to the hierarchy problem. But our failure to understand the cosmological constant, from the beginning, raised the nagging worry that there might be other explanations of apparent fine tunings. String theory made low energy supersymmetry seem more plausible; string/M theory is replete with solutions with $N=1$ supersymmetry to all orders in various expansion parameters. But for many years, the failure to find isolated vacua (and again to understand the cosmological constant) left considerable uncertainty as to whether low energy supersymmetry might be an outcome of string theory.

For better or worse, we have, at present, only one concrete proposal to understand the apparent small value of the cosmological constant, $\Lambda$, what Weinberg has dubbed the "weak anthropic principle" [1] (more recently, Weinberg's specific implementation has been called the "structure principle" in [2]). This is the idea that there is a vast "landscape" of possible stable or metastable ground states of the system, in which the cosmological constant takes on a "discretuum" of values. Weinberg (and subsequently [3]) argued that while different regions/periods of the universe might exist in different states, only those with sufficiently small $\Lambda$ would lead to cosmological formation of complicated structure.

Various suggestions as to how a landscape might arise have been made through the years. The most detailed and plausible implementation of this program to date has been provided by KKLT [5]. These authors studied compactifications of string theory on Calabi-Yau spaces with fluxes. They noted that the presence of fluxes typically fixes the complex structure moduli, and argued that generically various effects would fix the Kahler moduli as well (further studies of the stabilization of Kahler moduli appear in [6]). In the simplest version, the states are supersymmetric, with AdS supersymmetry. However, they noted that states with broken supersymmetry are likely to exist, at the same level of analysis.

There has been much handwringing about the question of whether such a picture can in any sense be viewed as science. It is not clear, for example, that a priori predictions (and falsification) are possible. Douglas has advocated a statistical analysis of string vacuum states, and has initiated such an effort with various collaborators [12, 11] as has Kachru and collaborators $[7,8]$. In [9], some of the present authors considered a number of issues which the landscape must confront. Some cautionary notes about the very existence of the flux discretuum were advanced. But accepting the picture suggested by effective field theory at face value, it was argued 
that the flux discretuum might well be falsifiable, and at the same time, might be predictive. It was stressed that within the flux discretuum, all of the parameters of low energy physics - the particle content, gauge groups, coupling constants, etc., vary as one moves around in the landscape. As a result, the question which Weinberg raised is enlarged and sharpened. We might imagine, for example, that the hierarchy has the value it has because only if the weak scale is close to its current value, can the sort of complex astrophysics and chemistry required for life arise. This sort of weak anthropic reasoning might also explain the values of other constants, such as the gauge couplings. But it was also noted that there are many parameters of low energy physics which do not have any obvious implication for the existence of observers. These should either be random variables, typical of the distribution, or they should be determined by some underlying principles. While the sharpest potential conflict is provided by the small value of $\theta_{\mathrm{QCD}}$, the masses and mixings of the heavier quarks, the weak gauge group, and some cosmological parameters are also puzzling. Possible problems with symmetry explanations and light moduli were discussed.

But if one closes ones eyes to this set of worries, there is an interesting possible prediction: low energy supersymmetry with visible sector superpartner masses at or near the weak scale. Given that SUSY effective field theories are inherent to the KKLT construction, one might imagine that this is the easiest - and most urgent - question to address. In the spirit of the program which has been pursued by Douglas et al and Kachru et al, one must ask whether the vast majority of states with, say, small cosmological constant and a hierarchy between the weak and Planck scales might have supersymmetry broken at comparatively low energy. A tentative argument - scenario might be a better word - along these lines was put forward in [9]. This scenario has been criticized in $[15,13,14]$. In this note, we sharpen the argument that low energy SUSY is a likely outcome of the landscape. Reviewing studies of the landscape done to date, we outline several scenarios for more detailed phenomenologies. These include a rather standard hidden sector supergravity scenario and a special case of the proposal of of [2] (which is in fact quite close to a proposal put forward long ago in [16]). But we argue that with some rather mild assumptions about discrete symmetries in the landscape, supersymmetry breaking at the lowest possible scales is favored (i.e. something like gauge mediation).

In the next section, we briefly review the KKLT analysis, focusing on the nature of the approximation, and on distinguishing supersymmetric and non-supersymmetric states. In particular, we will explain the sense in which supersymmetry, if unbroken at lowest order in a certain small parameter, remains unbroken to all orders. Supersymmetry breaking, then, must be a feature of the non-perturbative dynamics of the light fields. This implies, we will argue, 
a distribution of supersymmetry breaking scales which is roughly flat on a logarithmic scale. In the following section, we will discuss selection issues. We argue, quite generally, that quantities which are not subject to anthropic constraints must be typical of the distributions. We review why quantities such as $\theta_{\mathrm{QCD}}$ do not seem typical of the flux discretuum. But laying this concern aside, we put forward a pragmatic approach to the anthropic principle. We will argue that there are a number of quantities which could well be anthropically constrained the cosmological constant, the dark energy density, the baryon to photon ratio, the gauge couplings, the light quark masses, and others. It is probably beyond our present capabilities in science to claim that observers exist only at one (or a few) points in this parameter space. Instead, as we explain in section three, we will take the point of view that nature has done the analysis correctly. We will simply require that the cosmological constant, the weak scale, the dark energy density and so on lie near their observed values (we refer to this as the "Pragmatic Anthropic Principle"). The question of predicting supersymmetry is then the question: are the vast majority of states which satisfy these constraints approximately supersymmetric (in the sense of low energy supersymmetry).

In the fourth section, we will impose the constraints on the cosmological constant and weak scales. We will see that in the KKLT version of the landscape, small cosmological constant and dynamical supersymmetry breaking leads to a distribution of supersymmetry breaking scales that is roughly uniform on a logarithmic scale. Low energy supersymmetry is not particularly favored, but neither is it disfavored. However, requiring also that the Higgs mass be near its observed value strongly disfavors supersymmetry breaking at scales larger than the intermediate scale, provided the $\mu$ term vanishes in the leading approximation. We argue that the cost of a vanishing $\mu$ term might not be severe. But we also conjecture that there is likely to be a large set of states in which, in the leading approximation, supersymmetry is unbroken with $W=0$, as a result of discrete symmetries. Dynamical supersymmetry breaking in some of these states leads to a quite different distribution of cosmological constants. If such symmetries are not too rare on the landscape, supersymmetry breaking at the lowest possible energies is overwhelmingly favored.

Section five is devoted to enumerating pathways to phenomenology based on these observations. Very low energy supersymmetry breaking is suggestive of gauge mediation. But the higher scale breaking which seems to result from the KKLT vacua also suggests interesting possibilities. We note that the KKLT version of the landscape has a cosmological moduli problem, if the supersymmetry breaking scale is low. The Kahler moduli are fixed in the KKLT story, but in states with small cosmological constant and small supersymmetry breaking scale, their 
masses are comparable to $m_{3 / 2}$. Obtaining an acceptable dark matter spectrum then argues against a very low scale for supersymmetry breaking (less than the intermediate scale). In this version of the landscape, we suggest, the most plausible explanation for the dark matter density is that the moduli are heavy, with masses of order $100 \mathrm{TeV}$. We review an old argument why in a large class of models, dynamical supersymmetry breaking at intermediate scales leads to an anomaly mediated spectrum for gauginos (but not squarks and sleptons) [16]. This is, in fact, one of the scenarios advocated recently by Arkani-Hamed and Dimopoulos [2]. Another class of models leads to a more conventional picture (but with the usual problems of flavor changing processes).

Up to this point, we have contrasted supersymmetric states with non-supersymmetric states without any special features (what we might call "randomly broken supersymmetry" (RBSs)). References $[13,8]$ suggest that many of the flux vacua lie near conifold points. This raises the prospect that hierarchies may arise through warping. Reasons to think that warping or low energy supersymmetry might predominate are enumerated. We note, in particular, that stationary points with low energy supersymmetry are necessarily local minima of the moduli potential, while this is not the case for non-supersymmetric conifolds.

In the conclusions, we try to enumerate a set of questions which, if answered, would settle the question of whether low energy supersymmetry breaking, warping, or RBSS might emerge from the landscape. We explain that many of these questions are accessible. If low energy supersymmetry is favored, it may even be possible to determine which if any of the pathways we have listed is favored. We stress that not only may the landscape be predictive, it may be falsifiable. We also stress that within the landscape, conventional notions of naturalness are sharpened, not abrogated.

\section{Supersymmetric and Non-Supersymmetric Vacua in the Flux Discretuum}

We will take the KKLT analysis as our model for determining parameters in the discretuum. Clearly we do not understand the theory well enough to say that the KKLT states exist, much less that they are typical. But this gives us a concrete framework in which to work, and to explore possible scenarios.

KKLT imagine self-consistently fixing the values of the moduli. They first integrate out Kaluza-Klein modes, and study an effective action for complex structure and Kahler moduli. 
They then show that the superpotential can fix all of the complex structure moduli. Integrating out these fields, leaves an effective action for the Kahler moduli (which we denote generically by $R$ ). This analysis is reliable provided that the compactification radii are large. A crucial parameter in this action is $W_{o}$, the expectation value of the superpotential. They argue that non-perturbative effects in this action in $R$ (i.e. exponentially small for large $R$ ) will fix the remaining Kahler moduli, $R \sim-\ln \left(W_{o}\right)$. So the approximation may be reliable precisely in the limit of small $W_{o}$, which we will see is the regime in which low energy supersymmetry breaking may be important.

In the simplest version of this story, supersymmetry is unbroken at this stage. The small parameter is $W_{o}$; it determines the radii and the masses of the Kahler moduli. Large values of fluxes can also serve as small parameters. In the discretuum, $W_{o}$ can be argued to be distributed roughly uniformly as a complex variable $[5,13]$. Corrections to this approximation will not generate SUSY-breaking $F$ or $D$ terms for the moduli. This follows from an argument given long ago by Witten [26] (suitably generalized to AdS spaces): in order that supersymmetry be broken spontaneously, it is necessary that there exist a massless particle which can serve as the Goldstino (longitudinal mode of the gravitino). If supersymmetry is broken spontaneously, this must be due to the dynamics of other light fields, as in field theories which exhibit dynamical supersymmetry breaking.

KKLT went on to consider some possible mechanisms for supersymmetry breaking, in particular by considering configurations with anti D3 branes. But for our purposes, what is important here is that there is no reason to think there are many more non-supersymmetric than supersymmetric vacua at this level of approximation.

Within theories in which supersymmetry is broken at lowest order, Douglas has argued that supersymmetry breaking is likely to occur at the shortest distance scales [14]. A similar argument has been made by Susskind [15]. Indeed, for such states, it seems likely that the overwhelming number of states have supersymmetry broken at the Planck scale, so there are simply far more states with small cosmological constant and large SUSY breaking than with small SUSY breaking.

But as long as the number of supersymmetric states is at least comparable to the number of non-supersymmetric states, we would argue that these are by far the most important. Our experience with supersymmetric field theories would suggest that dynamical supersymmetry breaking is common. Indeed, if the landscape has anything to do with nature, chiral gauge theories must occur frequently, and such theories often break supersymmetry. Supersymmetry 
breaking scales in these states are likely to be distributed uniformly on a log scale. We will argue shortly that this dynamical breaking is likely to provide far more states that satisfy our anthropic requirements than the (random) non-supersymmetric ones with tree-level breaking.

Of course, there could be other constructions of some type which produce overwhelmingly more states with broken supersymmetry. Fortunately, these are questions we might realistically hope to answer over time. For now, our working assumption will be that this is not the case.

\section{A Pragmatic Approach to the Anthropic Principle}

The anthropic principle, whatever ones attitude towards it, is difficult to implement in practice. Even the seemingly simple question of the cosmological constant raises troubling issues. For example, if one only requires the formation of structure, and if one allows the dark matter density or the baryon to photon ratio or the amplitude of initial perturbations to vary, there is a huge range of allowed values of the cosmological constant [17]. We will see quite explicitly in our subsequent discussion that these quantities can all vary wildly in the landscape. It is possible that more complicated considerations involving the detailed nature of galaxies and stars sufficiently constrain the parameter space that all of these quantities are fixed. Similar statements apply to various microscopic quantities, such as the QCD scale, the light quark masses, and so on.

Our approach to selection will be pragmatic (we will refer to this as the Pragmatic Anthropic Principle, PAP). We will assume that nature has implemented the anthropic principle correctly, and that all quantities which might plausibly be anthropic are fixed by some set of considerations to be close to their present values (say within $10 \%$ or so). Specifically, we will assume that the following are fixed:

1. The cosmological constant

2. The ratio of the weak scale to the Planck scale

3. The QCD scale and the gauge couplings

4. The dark matter density

5. The baryon to photon ratio

6. The primordial fluctuation spectrum 
The question is: granted this set of assumptions, is it possible to make predictions in the landscape; equivalently, is the landscape falsifiable.

There are, as we have argued elsewhere, a number of quantities for which it is difficult to conceive of anthropic arguments. One example is the QCD $\theta$ parameter [18]. In the KKLT version of the landscape, it is necessary that the small value of $\theta_{\mathrm{QCD}}$ somehow be correlated with some other anthropic requirement. Perhaps, for example, it is necessary that axions provide the dark matter. This might explain the presence of a light axion. Perhaps, for example, some modulus is not fixed as in KKLT, but instead is only stabilized by low energy dynamics, leaving a light axion; this might be selected for by cosmological considerations. Another possibility, is that an $R$-axion common to many models of dynamical supersymmetry breaking acts as the QCD axion. By itself, neither of these ideas is adequate to explain the smallness of $\theta_{\mathrm{QCD}}$, however. Additional discrete symmetries, or something else, are probably required. It is quite possible that the whole landscape picture might be falsified on such grounds. For now, we will assume that there is a solution of this and similar problems. We will focus, instead, on supersymmetry. Since supersymmetry is an essential feature of the KKLT construction (and most string constructions), it would seem that the question of low energy supersymmetry would be the most straightforward to settle. Douglas and Susskind have argued that there is no prediction of low energy supersymmetry in the landscape. ${ }^{1}$ If this is the case, it is quite disappointing. It would suggest that the landscape may make no distinctive verifiable predictions, and that we have no theoretical or experimental access to the questions we have long viewed as fundamental in particle physics. But as we have explained, this argument is based on looking at a set of states which are not likely to be particularly important (but they are, as Douglas has stressed, the states for which the most straightforward analysis is currently possible).

\section{Is Supersymmetry a Feature of the Landscape?}

\subsection{Supersymmetry and Discrete Symmetries}

The landscape, whether it leads to supersymmetry or not, almost certainly requires discrete symmetries to be consistent with the requirements we have enumerated above. For example, the authors of [19] list a minimal extension of the Standard Model to account for the requirements given above. This minimal model includes a light, stable scalar which serves as the dark matter.

\footnotetext{
${ }^{1}$ More recently, both authors have realized that low energy dynamical breaking might allow such a prediction.
} 
This particle is assumed stable due to discrete symmetries (and it is light through tuning). An alternative dark matter candidate would be an axion, but if the axion is to be sufficiently light without extraordinary fine tuning (and, again, it is not clear what anthropic consideration would require this), one needs elaborate discrete symmetries. Other features of the low energy theory probably require symmetries as well.

In the case of supersymmetry, the need for discrete symmetries is at least as urgent. As noted in [9], anthropic considerations do not account for the long life of the proton, so something analogous to $R$-parity is probably required. Discrete symmetries might also be required to understand the smallness of the $\mu$ term (it would be interesting to know whether, as in weakly coupled strings, light fields arise in the flux discretuum without such symmetries). As we will discuss later, in warped scenarios, the need for discrete symmetries may be more urgent.

Discrete symmetries are ubiquitous in weakly coupled strings. Typical toroidal or CalabiYau compactifications have large discrete symmetry groups at points in the moduli space. It is clear that they can also arise in the landscape, but that there is some price to be paid; in the flux vacua, some number of fluxes must vanish. In [9], some simplified models were examined in order to see what fraction of states might be expected to possess useful discrete symmetries. The results were not conclusive, and the models not sufficiently realistic to make a reliable assessment. Kachru and collaborators are currently examining this question in more realistic models, and tentatively conclude that the price is not high (i.e. not a huge exponential factor) $[20]$.

\subsection{Is Supersymmetry Favored}

In [9], a scenario was outlined in which low energy supersymmetry might emerge as a prediction of the landscape. Here we will consider this type of argument in more detail. As stressed above, our focus will be on dynamical supersymmetry breaking in the supersymmetric states of KKLT. This was, in fact, the focus of [9]. We will see that the argument given there that a uniform distribution of $W_{o}$ along with the constraint of a small cosmological constant favors low energy supersymmetry is not correct. Instead, with these assumptions one predicts that the supersymmetry breaking scale has a flat distribution on a logarithmic scale.

We model dynamical supersymmetry breaking by supposing that the scale of supersymme-

try breaking is $\mu^{2}=e^{-8 \pi^{2} / g^{2}}$. In addition, we suppose that the distribution of $g^{2}$ is reasonably 
flat [13]. ${ }^{2}$ This is in fact the case for vacua near a conifold for which the distance from the conifold point is dual to a scale generated dynamically by dimensional transmutation $[13,8]$. We want to ask the probability that $\Lambda<\Lambda_{o}$ (to simplify, we will take the cosmological constant to be positive). We suppose that

$$
\Lambda=\mu^{4}-3\left|W_{o}\right|^{2}
$$

where $M_{p}=1$, and for the observed value of the vacuum energy $m_{3 / 2} \sim\left|W_{o}\right|$. Calling $F_{1}\left(\Lambda_{o}\right)$ the fraction of states with $\Lambda<\Lambda_{o}, P_{W}\left(W_{o}\right)$ the probability density for $W_{o}$, and $P_{g^{2}}\left(g^{2}\right)$ the probability density for the appropriate coupling, we have:

$$
\begin{array}{r}
F_{1}\left(\Lambda<\Lambda_{o}\right)=\int_{0}^{W_{\max }} d^{2} W_{o} P_{W}\left(W_{o}\right) \int_{\ln \left(\left|W_{o}\right|^{2}\right)}^{\ln \left(\left|W_{o}\right|^{2}+\Lambda_{o}\right)} d\left(g^{2}\right) P_{g^{2}}\left(g^{2}\right) \\
\approx \int_{0}^{W_{\max }} d^{2} W_{o} \frac{\Lambda_{o}}{\left|W_{o}\right|^{2}} P_{W}\left(W_{o}\right) \frac{1}{\ln W_{o}{ }^{2}} P_{g^{2}}\left(-1 / \ln \left(W_{o}\right)\right)
\end{array}
$$

So we see that if $P_{W}$ is reasonably flat near the origin, as is $P_{g^{2}}$ then this condition, by itself, predicts a distribution of supersymmetry breaking which is flat on a log scale. Note that the prefactor in the second line of (2) for the total fraction of vacua with vacuum energy $\Lambda<\Lambda_{o}$ is the vacuum energy $\Lambda_{o}$ itself. So the tuning associated with the observed value of the vacuum energy for this class of vacua with uniformaly distributed $W_{o}$ and dynamically broken supersymmetry is then roughly $\Lambda_{o} \sim 10^{-120}$ per decade.

Considering the question of the gauge hierarchy, however, does strongly disfavor very large values of $W_{o}$. Now, if the vacuum energy and weak scales are uncorrelated, we might expect that the fraction of states with fixed Higgs mass $m_{H}$ for $W_{o} \sim m_{3 / 2} \gg m_{H}$, would be suppressed by at least $\frac{m_{H}^{2}}{W_{o}^{2}}$ because of the requisite tuning of the Higgs potential. However, for $m_{H}^{2} M_{p} \lesssim$ $W_{o} \lesssim m_{H} M_{p}^{2}$ there is not necessarily any additional tuning since for any $W_{o}$ in this range there is in principle always some messenger sector which gives $m_{H}$ of order the electroweak scale. So if we call $F_{2}$ the fraction of states with a suitable $\Lambda$ and Higgs mass: $F_{2}\left(\Lambda<\Lambda_{o} ; 10 \mathrm{GeV}<\right.$ $\left.m_{H}<1 \mathrm{TeV}\right)$, say, this looks like:

$$
F_{2}\left(\Lambda<\Lambda_{o} ; 10 \mathrm{GeV}<m_{H}<1 \mathrm{TeV}\right) \sim\left\{\begin{array}{cl}
\Lambda_{o} \int_{W_{\text {int }}}^{W_{\max }} d^{2} W_{o} \frac{\mathrm{TeV}^{2}}{\left|W_{o}\right| 4} & W_{o}>W_{\mathrm{int}} \\
F_{1}\left(\Lambda<\Lambda_{o}\right) & W_{o}<W_{\mathrm{int}}
\end{array}\right.
$$

where $W_{\text {int }} \sim m_{3 / 2} M_{p}^{2}$ is the intermediate scale. So supersymmetry breaking well above the intermediate scale is strongly disfavored. Distinquishing a prefered supersymmetry breaking scale within the range $m_{H}^{2} M_{p} \lesssim W_{o} \lesssim m_{H} M_{p}^{2}$ requires answering the harder question of what type of messenger sectors are most common on this part of the landscape.

\footnotetext{
${ }^{2}$ We thank M. Douglas and L. Susskind for discussions of this point.
} 


\subsection{A Possibility for a Huge Enhancement}

In flux vacua constructions, the distribution of vacua is roughly uniform in $W_{o}$ once the moduli are fixed. From the point of view of obtaining models with a controlled approximation, a small non-zero $W$ is a virtue, because it leads to large values of the radii, and showing that some fraction of states had this property was one of the successes of KKLT. However, there may well be a substantial subset where $W_{o}$ vanishes, due to an unbroken, discrete $R$-symmetry (supersymmetry is still preserved). In such vacua, the Kahler moduli will generically be of order one and there are no obvious small parameters. But one expects that dynamical supersymmetry breaking in the low energy theory will sometimes generate both non-vanishing $F$ (and $D$ ) terms, as well as a non-vanishing $W$. If there are a discretuum of such states, then it is potentially much easier to cancel the cosmological constant than in the states with a flat distribution of $W_{o}$. Here it is helpful to put back the Planck scale, and note that if $\mu$ is the dynamical scale,

$$
|D W|^{2} \sim \mu^{4} ; 3|W|^{2} \sim \mu^{2} M_{p}^{2}
$$

These terms are of the same order if $\mu^{2} \sim m_{3 / 2} M_{p}$, i.e. for intermediate scale supersymmetry breaking (this is a point which T. Banks has stressed for many years). So, roughly speaking, among states with intermediate scale dynamical supersymmetry breaking and vanishing $W_{o}$, a fraction $\frac{\Lambda}{M_{i n t}^{4}}$ have suitable cosmological constant, as opposed to $\frac{\Lambda}{M_{p}^{4}}$. This is a huge advantage for these states, and may well win over the suppression required to obtain such a discrete symmetry.

But in fact, this sort of reasoning inevitably suggests that supersymmetry should be broken at the lowest possible scale. Suppose, for example, that in addition to the supersymmetry breaking sector, the theory contains another strongly interacting gauge theory which dynamically breaks the $R$-symmetry but not supersymmetry (as gluino condensation does). This will generate a constant superpotential, with a distribution essentially flat on a log scale. So if $\mu$ is the supersymmetry breaking scale, cancelling the cosmological constant favors low $\mu$ by $\mu^{-4}$, modulated by logarithms of $\mu$. This would tend to favor a phenomenology more like gauge mediation. For regions of the landscape with both $W$ and $D W$ generated dynamically the fraction of such states with small enough cosmological constant and appropriate weak scale could be as large as $10^{-60}$ for a supersymmetry breaking scale just above the weak scale, modulo any suppressions for additional symmetries, etc.

This would be one strong selection principle in favor of discrete $R$ symmetries, broken or partially broken only at scales well below the Planck scale. Apart from the question of the 
cosmological constant, this could also be relevant to proton decay, the $\mu$ problem, and dark matter.

\section{Paths to a Detailed Phenomenology}

\subsection{Gauge Mediation}

In the previous section, we have seen that supersymmetry breaking at the lowest possible energies (consistent, presumably, with various selection criteria and also the existence of an adequate density of states) is likely to be favored in the landscape. If this is actually the case, then something like gauge mediation seems the most likely outcome. This is in some sense a positive and exciting result, but it raises concerns. It is positive in that it provides the simplest explanation of how problems of flavor might be resolved in the landscape context. But it is troubling because gauge mediated scenarios at this stage tend to be somewhat tuned. One question is whether some selection criteria such as the ones listed in section 3 might explain some of this tuning when convoluted with features of the distribution of states on the landscape. We will not provide any answer to this question here.

In the rest of this section, we outline two other plausible scenarios for supersymmetry phenomenology. The first one has gauginos in the TeV energy range, but scalars at 100's of $\mathrm{TeV}$ or more (similar to one of the proposals in [2]). The second is a more conventional supergravity scenario. We should stress that it is possible that the landscape, as it is better understood, will make predictions which one can reliably say are in gross conflict or agreement with experiment.

\subsection{KKLT and the Moduli Problem}

If we take literally the construction outlined by KKLT, then coupled with a prediction of low energy supersymmetry, we also encounter a moduli problem. In the KKLT construction, the Kahler moduli (radii, etc.) are of order:

$$
R \approx-c \ln \left(W_{o}\right)
$$

and their masses are of order $W_{o}$. So the masses of the complex structure moduli, in this class of models are of order $m_{3 / 2}$. Such models will then, potentially, have a moduli problem. If $m_{3 / 2}$ is very small, as in low energy gauge mediation, such moduli would be particularly problematic. 
The PAP is likely to heavily disfavor such states ${ }^{3}$. If $m_{3 / 2}$ is of order $\mathrm{TeV}$, one has a more conventional moduli problem; this moduli problem would be solved if the moduli masses were $100 \mathrm{TeV}$ or so. We will see in the next subsection how such a picture might plausibly emerge from the landscape, and lead to an interesting phenomenology.

On the other hand, KKLT's requirement of large $R$ came, in part, from requiring calculability. But this is not something that nature requires, and we might imagine that, for generic superpotentials, the Kahler moduli will also be fixed at small radii and large mass. In particular, KKLT took the superpotential to include only a single exponential in these radii, but this is unlikely to be the complete superpotential. In the radii are small and masses are large, there would be no moduli problem. As we will discuss below, this leads to more conventional supergravity scenarios (with their potential flavor problems).

\subsection{A Higher Energy Scenario}

In this section, we will again take the KKLT construction literally. As we just noted, these vacua potentially suffer from a moduli problem. The Kahler moduli, in particular, have masses of order $W_{o}$.

In the context of "gravity mediation", the question of obtaining intermediate scale breaking dynamically was first addressed in [21]. These authors noted that one could readily couple a theory exhibiting DSB to supergravity, and obtain masses for scalars. At that time, the known models of DSB did not contain singlet fields, so it was is difficult to obtain gaugino masses. Subsequently, it was realized that anomaly mediated contributions to gaugino masses [16] would arise at one loop, but it was argued that such models would be finely tuned, given the experimental constraints on the gaugino masses and the value of the weak scale. It was also subsequently recognized that it is possible to construct models of dynamical supersymmetry breaking with singlet fields [27]. However, theories without singlets suggest a definite phenomenology which we briefly describe now; those with singlets allow an alternative which will be discussed in the next subsection.

In the landscape, the apparent tuning associated with heavy scalars which was previously so troubling is not relevant if the density of states is higher for a larger mass scale, or if selection criteria strongly favor the apparently tuned states.

\footnotetext{
${ }^{3}$ We certainly don't know how to treat this problem completely, but requiring, for example, that the initial values of the fields be sufficiently small that the moduli don't dominate the energy before the usual time of matter-radiation equality could easily cost a factor of $10^{12}$.
} 
In the present context, the most relevant selection criteria may well to be cosmological. Consider the questions of dark matter and the baryon asymmetry. As Moroi and Randall have stressed, in a situation like this, the LSP is likely to be the wino [22]. We have noted that there is probably a period of moduli domination; but neutralino dark matter can readily be produced in the decays of moduli [23]. A cosmology consistent with observation (but not necessarily forced by anthropic considerations) has the moduli and gravitino at $100 \mathrm{TeV}$ or so in order to obtain standard nucleosynethesis, with anomaly mediated gaugino masses in the few hundred GeV range. Baryons could be produced by the AD mechanism [24]. This requires that some moduli in fact carry baryon number. Baryons might also be produced by baryon violating processes in the decays of the moduli [25]. The moduli decays and the AD process both introduce several new parameters into the story. It is plausible that this structure, within the framework of the PAP, might actually favor visible sector supersymmetry breaking (i.e. scalar and gravitino masses) at the $100 \mathrm{TeV}$ scale. But note that scalars this heavy require a tuning of order at least roughly $10^{-6}$ in the Higgs potential in order to obtain acceptable electroweak symmetry breaking. So it is quite possible that the landscape favors a lower scale, again raising an opportunity to falsify the picture. For the moment, we can at least say that the facts of nucleosynthesis are consistent with such a spectrum.

We should note that there are further opportunities to falsify this scenario. The $100 \mathrm{TeV}$ scale is not quite high enough scale to completely solve all the flavor problems of supersymmetric theories (though it does solve the CP conserving problems). Perhaps there is some combination of anthropic arguments which forces the scale higher, or imposes approximate symmetries which adequately suppress various flavor violating processes. But it is also possible that there are not, and that this scenario within the landscape can be ruled out.

\subsection{A More Conventional Scenario}

It has been known for some time that one can construct models with gauge singlets which dynamically break supersymmetry even at the renormalizable level [27]. The couplings of these singlets are restricted. If this arises from discrete symmetries, gaugino masses may be suppressed, and we will be back to the picture of the previous subsection. But the required structure of couplings might arise because the singlets are approximate moduli, or perhaps some couplings are simply small, again through selection effects (this might not be particularly costly; the couplings don't need to be extraordinarily small). In this case, gaugino masses could be of the same order as scalar masses, leading to a more conventional supergravity phenomenology. 
In this case, however, the problems of flavor are potentially far more severe, as are the moduli problems. So again, there are opportunities to falsify this scenario within the landscape.

\section{Conifolds and Warping}

We have seen that low energy supersymmetry is likely to win over random non-supersymmetric configurations in the landscape. It has been noted, however, that generically flux vacua may arise near conifold points $[13,8]$. This may provide an alternative to supersymmetry to obtain hierarchies [28].

In fact, it would appear that one pays roughly the same price for such a hierarchy near a conifold point as one pays for dynamical supersymmetry breaking with a uniform distribution of $W_{o} \neq 0$ : first, a factor of $10^{-120}$ to explain the smallness of $\Lambda$, second, an order one factor to explain the gauge hierarchy, and third, some factor to obtain discrete symmetries, in order to suppress proton decay, obtain dark matter, etc.

One issue which may favor supersymmetry concerns the question of unstable (tachyonic) directions in the moduli mass matrix. In approximately supersymmetric vacua with $W_{o} \ll$ $1, \Lambda \ll\left|W_{o}\right|^{2}$, the moduli generically have large, positive mass-squared. In contrast, in the overwhelming majority of non-supersymmetric stationary points of the effective potential with large $W_{o}$ and small cosmological constant, one expects tachyons. We have seen, however, that if $W_{o}$ is zero at tree-level, then supersymmetry wins by an overwhelming amount. So it should be possible to settle the question of whether supersymmetry or non-supersymmetric conifolds are favored.

It is difficult to be quite so specific about the phenomenological issuses here as in the supersymmetric case. Proton decay is potentially a serious problem. If the energy scale of the Standard Model arises entirely through warping, it is necessary, most urgently, to understand why baryon number violating operators up to very high dimension are suppressed. One could imagine that this occurs through discrete symmetries, and that vacua with a high degree of symmetry are selected by anthropic considerations. However, anthropic selection probably does not require that the proton lifetime be nearly as large as it is observed to be. In other words, to be compatible with the current limit on the proton lifetime, it is necessary to suppress operators through dimension 13 or so; on the other hand, if the anthropic limit on the proton lifetime is

$10^{16}$ years, it is only necessary to suppress operators through dimension 11 . One could imagine that there would be many more vacua capable of suppressing the latter set of operators than 
the former. So typical, anthropically selected vacua might well be inconsistent with observation. Other proposed explanations of the long proton lifetime face similar potential difficulties.

This is in contrast to the situation in supersymmetric vacua, with at least an unbroken $R$-parity where it is necessary to suppress a range of dimension five operators, but already dimension six operators are not problematic.

This is clearly an issue which requires further study. It could well be that there are simply many more conifold type theories, with suitable properties, than supersymmetric theories, or vice versa; it could also be that some cosmological considerations favor one set or the other. For example, we have advanced a possible scenario in which the SUSY breaking scale might be larger than a $\mathrm{TeV}$, in order to allow suitable structure formation in the universe. This argument was not compelling; more knowledge about these types of vacua is necessary. Similarly, in the conifold case, it is not clear, at first sight, why we couldn't obtain a suitable dark matter density if the scale was one $\mathrm{TeV}$. In this case, flavor changing processes would likely falsify this possibility. However, it might be easier to suppress proton decay with a higher scale, or perhaps for some not quite so obvious reason it is easier to make a suitable dark matter density with a higher scale. These questions are difficult to resolve without much more detailed information about the distributions.

Finally, we should note in the original KKLT story, the breaking of supersymmetry by anti D3 branes located down the throat is perhaps to be thought of as the dual of a picture of field-theoretic dynamical supersymmetry breaking, and so fits in with the discussion of the previous section. ${ }^{4}$.

It is also possible to consider scenarios with large flat extra dimensions without warping. In such scenarios it might be possible to imagine that the fundamental scale is just above the weak scale, leading to a fraction of such such states with sufficiently small cosmological constant as large as $10^{-60}$. However, avoiding an additional price for the small four-dimensional Planck scale would require natural mechanisms for obtaining large volume (this might be obtained with two large dimensions). And the problem of proton stability in generic vacua of this type also must be addressed, just as in the case with warping.

\footnotetext{
${ }^{4}$ We thank L. Susskind for discussions of this point
} 


\section{Conclusions}

We have advanced a scenario, which we view as quite plausible, in which the landscape predicts low energy supersymmetry, possibly of a quite specific form. But we should stress that this is only a scenario, and we have described ways in which supersymmetry might not emerge as a prediction. We have noted that supersymmetry may be too costly: there might be far fewer states with the requisite discrete symmetries to prevent proton decay then there are nonsupersymmetric states. A second possibility, as we discussed in the previous section, is that warped geometries are sufficiently more numerous than states with dynamical supersymmetry breaking. Some pitfalls with the conifold scenario were noted, but it is not yet possible to argue decisively for the relative importance of one or another of these classes of vacua on the landscape.

If the following questions were answered, one could establish that supersymmetry is or is not a likely outcome of the landscape:

1. Are there, in the leading tree-level approximation, exponentially more non-supersymmetric than supersymmetric vacua? We have indicated that the answer to this question is likely to be no, but we certainly cannot claim to have proven such a statement. This would favor low energy supersymmetry.

2. What is the price of discrete symmetries? In particular, we need to compare the cost of suppressing proton decay and (if necessary) obtaining a small $\mu$ term with the price of light Higgs without supersymmetry $\left(10^{-36}\right.$ or so), times the price of obtaining a stable, light dark matter particle (unknown, but probably not less than $10^{-36}$ ), times the other tunings required to obtain an acceptable cosmology.

3. Is there a huge price for obtaining theories with low energy dynamical supersymmetry breaking? Given the presumption that one can obtain a landscape of models with complicated gauge groups and chiral matter, it is hard to imagine that the price is enormous (in landscape terms). A part in a billion, for example, would likely lead to a prediction of low energy supersymmetry.

4. Are unbroken discrete $R$-symmetries at the high scale common? If so, $\langle W\rangle$ must be generated dynamically at low energies in such vacua. In this case, we have seen that SUSY breaking at the lowest possible scale may be favored. 
5. Within the present knowledge of the landscape, non-supersymmetric conifolds appear to be the most promising alternative to low energy supersymmetry. What is the relative abundance of such states compared to supersymmetric states?

Related to the question of discrete symmetries, it would be interesting to know whether in supersymmetric states in the landscape, one often obtains massless, vector-like states (i.e. whether one can obtain, as in weakly coupled string theory, a small $\mu$ term without symmetries).

Answering these questions would establish whether supersymmetric vacua, as opposed to random non-supersymmetric vacua, are far more likely to satisfy the anthropic constraints we have enumerated. If the price of vanishing $W_{o}$ is not large, we have seen that supersymmetry breaking at the lowest possible scale wins.

More detailed information about the statistics of the landscape could further narrow the possibilities for string phenomenology. In particular, we have seen that information about the numbers of supersymmetry breaking low energy theories of various types could lead to quite specific predictions.

There are various ways the theory could fail to predict supersymmetry. If, for example, $W_{o}$ is non-zero at leading tree-level approximation in the overwhelming number of states and one has to understand the smallness of the $\mu$ term as a consequence of tuning, this might eliminate any possible prediction of low energy supersymmetry. If it should turn out, with further exploration of the landscape, that there are vastly more non-supersymmetric than supersymmetric states, again low energy supersymmetry is not a likely outcome of string theory.

We should also stress that even in the landscape, ordinary notions of naturalness are likely to be relevant. For example, we have seen that in the intermediate scale scenario for supersymmetry breaking primordial nucleosynthesis forces gaugino masses into the $\mathrm{TeV}$ region, with scalars in the $100 \mathrm{TeV}$ range. This is certainly "tuned"; there are almost certainly far more states with light Higgs particles with supersymmetry broken at lower energies. We have suggested a way in which this mini hierarchy might arise from selection criteria of a cosmological nature. We leave for further work the question of tuning in the very low energy scenario, but note that in this case, in addition to cosmological concerns, the need to account for the $\mu$ and $B_{\mu}$ parameters might explain requisite tunings. We have also noted that for the conifold scenario, proton decay and flavor violation require coincidences not readily explained by anthropic reasoning. 
The questions in this list seem accessible to investigation. In the background, however, there lurk other questions: are these states real and accessible cosmologically? If they are, does the history of the universe prefer some states over others?

Acknowledgements: This work supported by the U.S. Department of Energy. We thank A. Aguirre, Tom Banks, Michael Douglas, Willy Fischler, Shamit Kachru, and Lenny Susskind for discussions and protecting us from worse blunders than those we may have committed above. The work of M.D. and E.G. was supported in part by the US Department of Energy. The work of S.T. was supported in part by the US National Science Foundation under grant PHY02-44728.

\section{References}

[1] S. Weinberg, Rev. Mod. Phys. 61, 1 (1989).

[2] N. Arkani-Hamed and S. Dimopoulos, arXiv:hep-th/0405159.

[3] J. Garriga and A. Vilenkin, Phys. Rev. D 61, 083502 (2000) [arXiv:astro-ph/9908115].

[4] S. Weinberg, arXiv:astro-ph/0005265.

[5] S. Kachru, R. Kallosh, A. Linde and S. P. Trivedi, Phys. Rev. D 68, 046005 (2003) [arXiv:hep-th/0301240].

[6] F. Denef, M. R. Douglas and B. Florea, JHEP 0406, 034 (2004) [arXiv:hep-th/0404257].

[7] A. Giryavets, S. Kachru, P. K. Tripathy and S. P. Trivedi, JHEP 0404, 003 (2004) [arXiv:hep-th/0312104].

[8] A. Giryavets, S. Kachru and P. K. Tripathy, arXiv:hep-th/0404243.

[9] T. Banks, M. Dine and E. Gorbatov, arXiv:hep-th/0309170.

[10] S. Ashok and M. R. Douglas, JHEP 0401, 060 (2004) JHEP 0401, 060 (2004) [arXiv:hepth/0307049].

[11] M. R. Douglas, arXiv:hep-ph/0401004.

[12] M. R. Douglas, JHEP 0305, 046 (2003) [arXiv:hep-th/0303194].

[13] F. Denef and M. R. Douglas, JHEP 0405, 072 (2004) [arXiv:hep-th/0404116].

[14] M. R. Douglas, arXiv:hep-th/0405279.

[15] L. Susskind, arXiv:hep-th/0405189.

[16] M. Dine and D. MacIntire, Phys. Rev. D 46, 2594 (1992) [arXiv:hep-ph/9205227]. 
[17] A. Aguirre, Phys. Rev. D 64, 083508 (2001) [arXiv:astro-ph/0106143].

[18] J. F. Donoghue, Phys. Rev. D 69, 106012 (2004) [Erratum-ibid. D 69, 129901 (2004)] [arXiv:hep-th/0310203].

[19] H. Davoudiasl, R. Kitano, T. Li and H. Murayama, arXiv:hep-ph/0405097.

[20] Shamit Kachru, private communication.

[21] I. Affleck, M. Dine and N. Seiberg, Nucl. Phys. B 256, 557 (1985).

[22] T. Moroi and L. Randall, Nucl. Phys. B 570, 455 (2000) [arXiv:hep-ph/9906527].

[23] L. Randall and S. Thomas, Nucl. Phys. B 449, 229 (1995) [arXiv:hep-ph/9407248].

[24] M. Dine, L. Randall and S. Thomas, Nucl. Phys. B 458, 291 (1996) [arXiv:hepph/9507453].

[25] T. Banks, D. B. Kaplan and A. E. Nelson, Phys. Rev. D 49, 779 (1994) [arXiv:hep$\mathrm{ph} / 9308292]$.

[26] E. Witten, Nucl. Phys. B 188, 513 (1981).

[27] K. A. Intriligator and S. Thomas, Nucl. Phys. B 473, 121 (1996) [arXiv:hep-th/9603158].

[28] S. B. Giddings, S. Kachru and J. Polchinski, Phys. Rev. D 66, 106006 (2002) [arXiv:hepth/0105097]. 